\title{
Acute cholangitis in a patient with Kluyvera cryocrescens bacteremia: A case report and literature review
}

\author{
Steven D. Kozusko*1, Paul Kolarsick², Ernest M. Ginalis² \\ ${ }^{1}$ University of Tennessee Health Science Center, Department of Plastic Surgery, Memphis, United States \\ ${ }^{2}$ Monmouth Medical Center, Department of General Surgery, Long Branch, United States
}

Received: August 7, 2016

DOI: $10.5430 /$ css.v2n4p46
Accepted: September 8, 2016

Online Published: September 12, 2016

URL: http://dx.doi.org/10.5430/css.v2n4p46

\begin{abstract}
Background: Originally thought to be benign organisms, there is increasing evidence that Kluyvera species harbor the potential to induce a multitude of pathologies. With increasing evidence implicating Kluyvera in various pathologies, including those of soft tissue and the biliary tract, it is important that both plastic and general surgeons are well informed of this organism and its potential manifestations.

Case presentation: A thirty-five years old man was admitted to Monmouth Medical Center with right upper quadrant (RUQ) and abdominal pain of acute onset. He was found to have Kluyvera cryocrescens bacteremia secondary to acute cholangitis. He was treated with a laparoscopic cholecystectomy and antibiotics. He made a full recovery.

Discussion: There are a multitude of manifestations of Kluyvera infection, including urinary tract infections, sepsis and bacteremia, diarrhea, soft tissue infection, cholecystitis, intra-abdominal abscess, pancreatitis, mediastinitis, and urethrorectal fistula. Early identification of this infection is important so that appropriate antibiotic coverage can be initiated.

Conclusions: We present the first documented case of acute cholangitis in a patient with Kluyvera cryocrescens bacteremia. The patient was successfully treated with a course of antibiotics and laparoscopic cholecystectomy.
\end{abstract}

Key Words: Kluyvera, Kluyvera cryocrescens, Kluyvera ascorbata, Cholangitis, Laparoscopic, Cholecystectomy, Plastic surgery, General surgery

\section{INTRODUCTION}

Originally thought to be benign organisms, there is increasing evidence that Kluyvera species harbor the potential to induce a multitude of pathologies. ${ }^{[1-5]}$ Kluyvera are Gram negative bacilli which can colonize the respiratory, gastrointestinal, and urinary tracts. These organisms are small, motile, and possess peritrichous flagella. This genus is similar to that of Enterobacteriaceae. ${ }^{[4]}$ Kluyvera are unique from Enterobacteriaceae in that the former utilize malonate and citrate.
Additionally, Kluyvera has a positive reaction in Moeller ornithine decarboxylase. ${ }^{[6]}$ Described as an organism that rarely causes opportunistic infections, Kluyvera can affect both immunocompromised and immunocompetent individuals. ${ }^{[7]}$ This species is widely distributed in nature, with reports of environmental sources including water, hospital sinks, sewage, animals, and milk. ${ }^{[5]}$ The four species of Kluyvera identified to date are cryocrescens, ascorbata, georgiana, and cochleae. In pediatric populations Kluyvera has

\footnotetext{
*Correspondence: Steven D. Kozusko; Email: skozusko@uthsc.edu; Address: University of Tennessee Health Science Center, Department of Plastic Surgery, Memphis, TN 38163, United States.
} 
been cultured from sources such as stool and cerebrospinal fluid. ${ }^{[7]}$

The total number of clinically significant Kluyvera infections published in 2005 was 41, with 21 being Kluyvera ascorbata and 8 from Kluyvera cryocrescens. ${ }^{[8]}$ The virulence conferred by Kluyvera may be from its lipopolysaccharide complex and surface antigens. ${ }^{[9]}$ Also contributing to its effects are host factors such as immune status.

Kluyvera is present in the human digestive system at low bacterial counts. ${ }^{[9]}$ This organism has the capability of infecting mucosal surfaces including the gastrointestinal and urinary tracts. Furthermore it is capable of infecting soft tissue. ${ }^{\text {9] }}$ Plastic surgeons should be informed of case reports involving sternal wounds, forearm infections, finger infections, and tenosynovitis requiring a skin flap. For general surgeons it is essential to know that Kluyvera has caused acute emphysematous cholecystitis, acute cholecystitis, and acute pancreatitis. We now present the first documented evidence of acute cholangitis caused by Kluyvera cryocrescens.

\section{CASE REPORT}

A thirty-five years old man was admitted to Monmouth Medical Center with right upper quadrant (RUQ) and abdominal pain of acute onset. Of note, the patient had a history of chronic alcoholism with a multiple drinks of liquor daily. On presentation the patient had tachypnea and fever measured at 101.1 degrees Fahrenheit. He was nauseous and actively vomiting. Labs drawn in the emergency department revealed hyperbilirubinemia with total and direct bilirubin of 6.0 milligram (mg) per deciliter (dl) and $4.0 \mathrm{mg} / \mathrm{dl}$, respectively. His liver function tests (LFTs) were mildly elevated with his aspartate transaminase (AST), alanine transaminase (ALT), and alkaline phosphatase (ALK) recorded at 217 units per liter, 406 units per liter, and 518 units per liter.

On further workup the patient had an absent acetaminophen level, ruling out toxicity as a cause of his elevated liver labs. A hepatitis panel was drawn and there were no antibodies suggestive of active or chronic infection. Additionally, a drug panel was completed and returned with negative results. His white blood cell (WBC) count was elevated to 16,300 WBCs per microliter (ml). His prothrombin time was noted to be 14.0 , and he had a normal international normalized ratio (INR). Blood cultures would grow the bacteria Kluyvera cryocrescens.

An ultrasound (US) of the liver and gallbladder depicted a distended gallbladder with cholelithiasis and a prominent common bile duct (CBD) of 6 millimeter (mm). Subsequent magnetic resonance cholangiopancreatography (MRCP) showed small filling defects within the CBD concerning for choledocholithiasis. An endoscopic retrograde cholangiopancreatography (ERCP) was completed 4 days later showing a normal caliber CBD with no choledocholithiasis. During this test a sphincterotomy was completed. With the working diagnosis of acute cholangitis and cholecystitis it was decided that the patient would undergo a cholecystectomy.
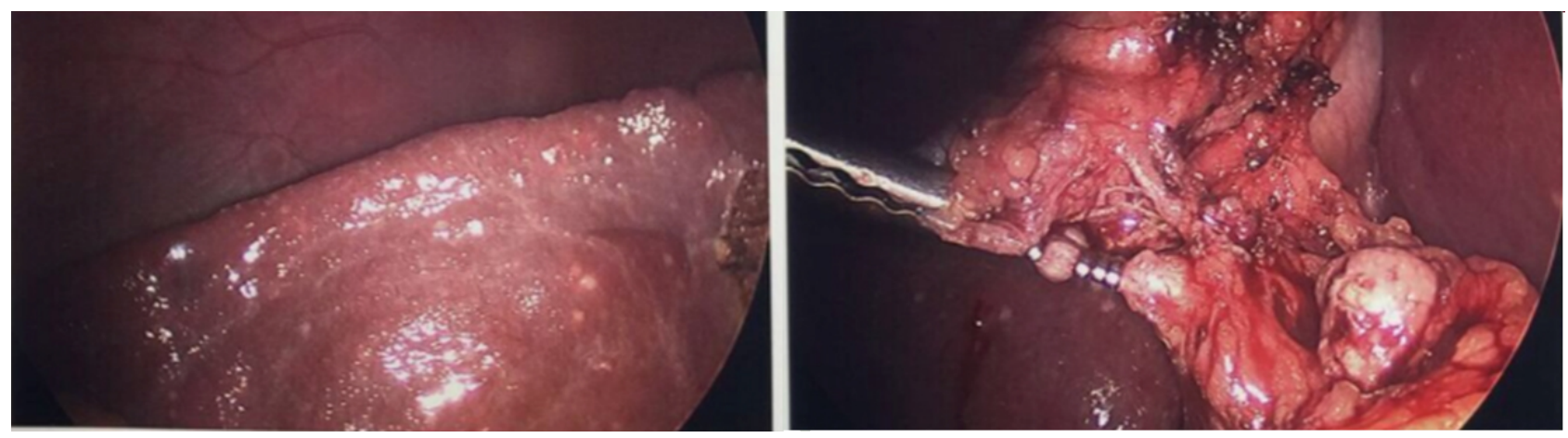

Figure 1. Intraoperative laparoscopic photographs depicting microabscesses

Intraoperatively the surgical team was surprised by the visual inspection of the liver. There were numerous $1 \mathrm{~mm}-2 \mathrm{~mm}$ white nodules throughout the liver (see Figure 1). These microabscesses were believed to be due to Kluyvera cryocrescens, as this was the bacteria causing bacteremia. The surgical team completed the laparoscopic cholecystectomy without complications. A biopsy of the liver was taken for pathology. The gallbladder pathology showed acute cholecys- titis. The liver pathology showed acute cholangitis, chronic hepatitis and bridging fibrosis/incomplete cirrhosis. The case was discussed at the pathology departmental conference due to its unique presentation.

Postoperatively the patient continued on levaquin and metronidazole. Repeat cultures were not obtained as the patient's temperature normalized and WBC dropped during the admission. Blood culture susceptibilities returned a strain 
of Kluyvera cryocrescens resistant to ampicillin, cefazolin, and cefuroxime. It had intermediate resistance to ceftriaxone. It was susceptible to cefepime, cefotaxime, ceftazidime, gentamicin, zosyn, tetracycline, and trimethoprim/sulfamethoxazole.

The patient followed up in the surgical clinic two weeks later. At that time he had no residual symptoms of RUQ or abdominal pain. He remained afebrile and reported that he felt back to his normal health status.

\section{Discussion}

There are a multitude of manifestations of Kluyvera infections reported in the literature (see Table 1). ${ }^{[1-17]}$ One unique case report from 1998 describes a diabetic patient who developed a finger blister that progressed to tenosynovitis requiring serial debridements and a skin flap. ${ }^{[16]}$ Another report found Kluyvera cryocrescens in a peritoneal cavity abscess in a patient undergoing peritoneal dialysis. ${ }^{[14]}$ This patient had end-stage renal disease and succumbed to her condition. Two cases of Kluyvera infection in recipients of solid organ transplants have been reported. ${ }^{[1]}$ One patient received a living donor liver transplantation and the other a deceased donor kidney transplant. The liver recipient grew out Kluyvera cryocrescens and the renal recipient's culture was Kluyvera ascorbata.

Table 1. Number of reported cases of Kluyvera infections

\begin{tabular}{lll}
\hline Clinical Manifestation & $\begin{array}{l}\text { Total } \\
\text { Kluyvera } \text { sp. }\end{array}$ & $\begin{array}{l}\text { Kluyvera } \\
\text { cryocrescens }\end{array}$ \\
\hline Urinary tract infection & 12 & 0 \\
Sepsis and bacteremia & 13 & 8 \\
Diarrhea & 7 & 0 \\
Soft tissue infection & 4 & 1 \\
Cholecystitis & 3 & 1 \\
Intra-abdominal abscess & 3 & 1 \\
Pancreatitis & 1 & 0 \\
Mediastinitis & 1 & 0 \\
Urethrorectal fistula & 1 & 0 \\
Liver abscess & 1 & 1 \\
Emphysematous gastritis & 1 & Not determined \\
\hline
\end{tabular}

Yoshino's study reported on indwelling devices with Kluyvera infection. ${ }^{[5]}$ Nine patients were found to have Kluyvera infection related to indwelling catheters including peripheral intravenous catheters and central venous catheters. The comorbidities of patients includes congestive heart failure, coronary artery disease, and interstitial lung disease. The most common antibiotic regimen used to treat these patients was cefazolin and gentamicin. The prognosis was good in these patients with 8 cured and 1 unknown outcome.

Infections have been reported in both adults and children. A ten-year-old pediatric female with chronic proteinuria was found to have a positive urine culture for Kluyvera cryocrescens ${ }^{[13]}$ In one preterm infant suspected to have necrotizing enterocolitis, blood cultures came back positive for Kluyvera cryocrescens which was susceptible to ceftazidime, amikacin, and ciprofloxacin. ${ }^{[1]}$ This patient's bacteremia was treated effectively with antibiotics.

Kluyvera cryocrescens has been documented in a specimen of gallbladder pus. ${ }^{[17]}$ Cultures from gallbladder fluid in one adult with acute pancreatitis also grew Kluyvera species. ${ }^{[6]}$ Another patient had acute appendicitis with a subsequent intraabdominal abscess positive for Kluyvera ${ }^{[4]}$ Our patient is the first reported and proven case of acute cholangitis due to Kluyvera species. The patient had bacteremia secondary to this infection, and the organism seeded the liver.

One important case series reported seven clinical manifestations of Kluyvera. The pathologies were manifested as a urinary tract infection, sepsis and bacteremia, diarrhea, soft tissue infection, cholecystitis, peritonitis and intra-abdominal abscess, pancreatitis, mediastinitis, and urethrorectal fistula. ${ }^{[8]}$ This may provide further evidence to the argument that Kluyvera is a commensal organism colonizing humans. In the patients with pancreatobiliary tract disease, three cultures were obtained from bile and one from blood. All patients with soft tissue infections recovered with antibiotic therapy.

Another literature review reported twenty-seven clinically significant Kluyvera infections. ${ }^{[9]}$ Their data included underlying conditions including cholelithiasis, bladder carcinoma, diabetes mellitus, colon adenocarcinoma, pyelonephritis, forearm soft tissue infection, finger soft tissue infection, tenosynovitis, lower urinary tract infection, hepatitis $\mathrm{B}$, hepatitis $\mathrm{C}$, cirrhosis, and chronic renal failure. The source of the organism was found in stool, gallbladder fluid, wound exudate, fistula drainage, blood, sternal wound, urine, and peritoneal fluid. Antibiotics used included trimethoprim/sulfamethoxazole, ampicillin with gentamicin, ciprofloxacin, cefotaxime, and ceftriaxone. Patient ages ranged from eleven months to seventy-five years.

Comorbidities seem to play a large factor in this organism's presentation. Malignancy, neutropenia, diabetes mellitus, chronic liver or renal disease, surgery, and trauma all may predispose the patient to an opportunistic infection. ${ }^{[9]}$ Our patient's chronic alcohol consumption likely predisposed him to bacteremia and acute cholangitis.

Early identification of this infection is important in order to initiate appropriate antibiotic therapy. However, there is no specific site that is favored by this organism and, eing a rare 
infection, it may not be intuitive which antibiotic is appropriate. ${ }^{[9]}$ In a study on antimicrobial susceptibility patterns of Kluyvera ascorbata and Kluyvera cryocrescens, natural resistance was found with many macrolides, lincosamides, streptogramins, glycopeptides, rifampin, fusidic acid, linezolid, penicillin $\mathrm{G}$, oxacillin, and amoxicillin. ${ }^{[15]}$ In the aforementioned study by Sarria et al., ${ }^{[9]}$ gentamicin was the only agent active against all strains of Kluyvera, but colistin, chloramphenicol, and kanamycin were active against more than ninety percent of strains. In their study they also reported agents most consistently active in vitro were $3^{\text {rd }}$ generation cephalosporins, fluoroquinolones, aminoglycosides, imipenem, chloramphenicol, and nitrofurantoin. Other data shows by production of beta-lactamases, resistance to ampicillin and $1^{\text {st }}$ and $2^{\text {nd }}$ generation cephalosporins is common. ${ }^{[7]}$ This study also recommended $3^{\text {rd }}$ generation cephalosporins, fluoroquinolones, aminoglycosides, tetracycline, aztreonam, and imipenem as treatment options. Once culture susceptibilities return the antibiotic(s) used to treat the patient should be tailored to those result.

Our patient received one week of levaquin and metronidazole. The culture susceptibilities showed ampicillin, cefazolin, and cefuroxime resistance. There was intermediate resistance to ceftriaxone. There was susceptibility to cefepime, cefotaxime, ceftazidime, gentamicin, zosyn, tetracycline, and bactrim. Consistent with data already published, Kluyvera remains susceptible to gentamicin.

\section{Conclusions}

We present the first documented case of acute cholangitis in a patient with Kluyvera cryocrescens bacteremia. The patient was successfully treated with a course of antibiotics and laparoscopic cholecystectomy.

\section{CONFLiCTS OF INTEREST DisClOSURE}

The authors declare they have no conflicts of interest.

\section{REFERENCES}

[1] Altun Koroglu O, Yalaz M, Ozalkaya E, et al. Kluyvera cryocrescens in a preterm infant. Jpn J Infect Dis. 2010 May; 63(3): 195-6. PMid: 20495273.

[2] Cheruvattath R, Balan V, Stewart R, et al. Kluyvera co-infection in two solid organ transplant recipients: an emerging pathogen or a colonizer bystander? Transpl Infect Dis. 2007 Mar; 9(1): 83-6. PMid: 17313481. http://dx.doi.org/10.1111/j.1399-3062. 2006.00198.x

[3] Carter JE, Evans TN. Clinically significant Kluyvera infections: a report of seven cases. Am J Clin Pathol. 2005 Mar; 123(3): 334-8. PMid: 15716228. http://dx.doi.org/10.1309/61XP4KTLJYW M5H35

[4] Eisenhut M, Hughes D, Ashworth M. Fatal emphysematous gastritis in a 2-year-old child with chronic renal failure. Pediatr Dev Pathol. 2004 Jul-Aug; 7(4): 414-6. PMid: 15383939. http://dx.doi.org /10.1007/s10024-003-7081-8

[5] Lin J, Chen C, Sui L, et al. Nosocomial outbreak of Kluyvera cryocrescens bacteremia. Infect Control Hosp Epidemiol. 2002; 23: 62-4. PMid: 11893149. http://dx.doi.org/10.1086/503456

[6] Luttrell RE, Rannick GA, Soto-Hernandez JL, et al. Kluyvera species soft tissue infection: case report and review. J Clin Microbiol. 1998 Dec; 26(12): 2650-1.

[7] Nicolosi D, Nicolosi VM, Cappellani A, et al. Antibiotic susceptibility profiles of uncommon bacterial species causing severe infections in Italy. J Chemother. 2009; 21: 253-60. PMid: 19567344. http://dx.doi.org/10.1179/joc.2009.21.3.253

[8] Ortega Calvo M, Delgado Zamora R, Fernandez Arance P, et al. Kluyvera cryocrescens: a positive urine culture in a young girl with persistent proteinuria. Acta Urol Esp. 1999 Jun; 23(6): 528-31. PMid: 10464962.
[9] Oteo J, Gomez-Garces JL, Alos JI. Acute cholecystitis and bacteremia caused by Kluyvera ascorbata in a cirrhotic patient. Clin Microbiol Infect. 1998 Feb; 4(2): 113-115. PMid: 11864301. http: //dx.doi.org/10.1111/j.1469-0691.1998.tb00370.x

[10] Sarria JC, Vidal AM, Kimbrough RC. $3^{\text {rd }}$. Infections caused by Kluyvera species in humans. Clin Infect Dis. 2001 Oct 1; 33(7): E6974. PMid: 11528588 . http://dx.doi.org/10.1086/322686

[11] Sezer MT, Gultekin M, Gunseren F, et al. A case of Kluyvera cryocrescens peritonitis in a CAPD patient. Perit Dial Int. 1996 May-Jun; 16(3): 326-7. PMid: 8761550.

[12] Stock I. Natural antimicrobial susceptibility patterns of Kluyvera ascorbata and Kluyvera cryocrescens strains and review of the clinical efficacy of antimicrobial agents used for the treatment of Kluyvera infections. J Chemother. 2005 Apr; 17(2): 143-60. PMid: 15920899. http://dx.doi.org/10.1179/joc.2005.17.2.143

[13] Toprak D, Soysal A, Turel O, et al. Hickman catheter related bacteremia with Kluyvera cryocrescens: a case report. Jpn J Infect Dis. 2008 May; 61(3): 229-30. PMid: 18503178.

[14] West BC, Vijayan H, Shekar R. Kluyvera cryocrescens finger infection: case report and review of eighteen Kluyvera infections in human beings. Diagn Microbiol Infect Dis. 1998 Nov; 32(3): 237-41. http://dx.doi.org/10.1016/S0732-8893(98) 00087-X

[15] Yoshino Y, Nakazawa S, Otani S, et al. Nosocomial bacteremia due to Kluyvera cryocrescens: case report and literature review. IDCases. 2016 Mar 4; 4: 24-6. http://dx.doi.org/10.1016/j.idcr. 20 16.02 .007

[16] Thaller R, Berlutti F, Thaller MC. A Kluyvera cryocrescens strain from a gall-bladder infection. Eur J Epidemiol. 1988 Mar; 4(1): 124 6. PMid: 3356228. http://dx.doi.org/10.1007/BF00152705

[17] Wong VK. Broviac catheter infection with Kluyvera cryocrescens: a case report. J Clin Microbiol. 1987 Jun; 25(6): 1115-6. PMid: 3597755 . 